\title{
A Remote Health Care System Combining a fall Down Alarm and Biomedical Signal Monitor System in an Android Smart-Phone
}

\author{
Ching-Sung Wang \\ Department of Electronic \\ Engineering Oriental Institute of \\ Technology, Taipei, Taiwan
}

\author{
Chien-Wei Liu \\ Department of Information \\ Management St. Mary's College, \\ Yilan, Taiwan
}

\author{
Teng-Hui Wang \\ Department of Computer Science \\ and Information Engineering, \\ National Taiwan University of \\ Science and Technology, Taipei \\ Taiwan
}

\begin{abstract}
First aid and immediate help are very important following an accident. The earlier the detection and treatment is carried out, the better the prognosis and chance of recovery of the patients. It is even more important when considering the elderly. Once the elderly have an accident, they not only physically injure their body, but also impair their mental and social ability, and may develop severe sequela. In the last few years, the continuously developed Android cell phone has been applied to many fields. Despite the nature of the GPS positioning system that the mobile phone currently uses, most applications used are SMS and file transfers. However, these biomedical measurement signals, passing through a transferring interface and uploading to the mobile, result the little really successful cases with the remote health care feasibility. This research will develop an Android cell phone which combines the functionality of an ECG, pulsimeter, SpO2, and BAD (Body Activity Detector) for real-time monitoring of the activity of a body. When an accident occurs, the signals go through Android smart phone, immediately notifying the remote ends and providing first time help.
\end{abstract}

Keywords-Health care; Biomedical signal; fall down alarm; Real-time; Android smart phone

\section{INTRODUCTION}

In 1991, the American Heart Association suggested, the "Chain of Survival" of early access, early CPR, early defibrillation, and early advanced care. It emphasized that immediately after cardiac arrest, early CPR in the first $4 \mathrm{~min}$. and early advance care within the first 8 min would have about a $43 \%$ chance of successful rescue rate, the alternative was lower than 20\%. Cumnius R.O.'s research points out that there is about a $7-10 \%$ decrease in rescue rate success per minute delayed after the patients' heart arrest [1].

In traumatic care, there is the so-called "golden hour" concept that refers to the first sixty minutes after the occurrence of multi-system trauma. It is widely believed that the victim's chances of survival are greatest if they receive definitive care in the operating room within the first hour after a severe injury (Committee on Trauma, 1993; Division of Trauma \& EMS, 1992).
For a cerebrovascular accident, it also highlights the "golden 3 hours", in that providing proper care in the first 3 hours after the accident can increase the chance of survival. Despite the kind of emergency, they focus on immediate response and properly provided early detection and early treatment.

Accidents are more significant for the elderly, morbid populations, and for those who have unstable life signs. According to studies, once an elderly person falls down, they would reduce their living activity [2][3], indirectly reducing their quality of life. Due to the habit of using an Android mobile phone, we suggest a tool set that is able to monitor the user's state of health [4][5][6][7]. It operates through Bluetooth SPP (Serial Port Profile) [8] to communicate with the mobile phone. When the BAD (Body Activity Device) detects an abnormal response, it will send an emergency signal to the Android mobile phone. After that, the Android mobile starts real-time biomedical signal monitoring, and efficiently transfers both the GPS and biomedical signals back to the server.

When an accident occurs, it can immediately and effectively provide relative rescue information by the mobile phone and Bluetooth, expecting that is able to improve first aid effect. In addition, it provides the senior population with an effective accident prevention guarantee, enabling them to avoid decreasing their life span because of an accident.

\section{System ARChiteCtURE}

This system is categorized into three main structures, client, server, and First Aid unit. The client includes BAD, a biomedical signal monitoring device (including ECG, $\mathrm{SpO}_{2}$ monitoring device) [9][10], and Android mobile phone with Bluetooth communication ability, When BAD detects an abnormal health response from the user, it will give the mobile a report by means of the Bluetooth. In the meantime, the operating biomedical device, in sending signals back, allows GPS coordination to take place. These messages would be sent back to server through the mobile phone's internet system. The server end is predominantly used to save the data, to analysis it, and to communicate with others. (Figure 1) 


\section{A. Client Side System}

This system includes $\mathrm{BAD}, \mathrm{ECG}, \mathrm{SpO}_{2}$, and Android mobile phones. They communicate with each other by means of the Bluetooth:

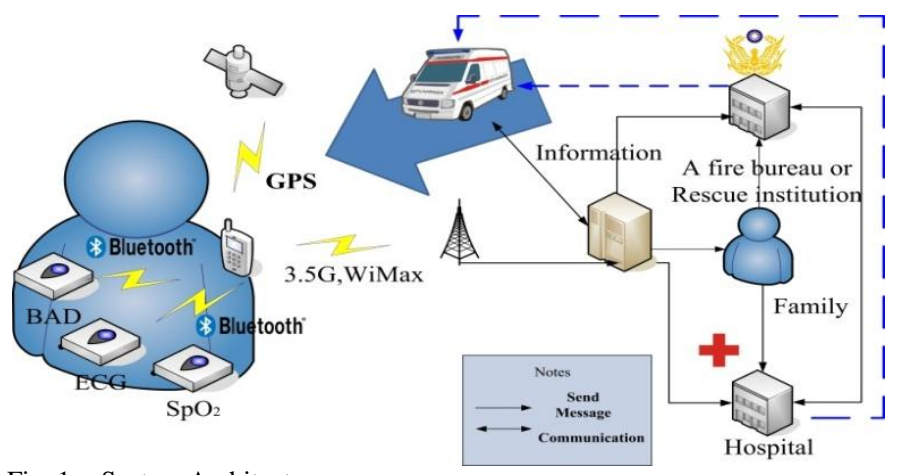

Fig. 1. System Architecture

1) BAD: The main function is to monitor the unusual, and the phone immediately sends such messages to the monitoring server through the public mobile network.

2) Biomedical signal detector: Uses the already set-up electric circuit and device to detect biomedical signals. After obtaining the signals, it digitalizes them and communicates with the mobile phone to do transferring the device includes ECG and $\mathrm{SpO}_{2}$ monitoring.

3) Android smart phone: Uses Android 2x version [11] smart new mobile technology as a communicating host. It carries a GPS coordinating device and a 3.5 wireless communication system. When an abnormal signal is detected, it immediately sends the user's address and signal back to the server for processing.

\section{B. Server Side System}

The server end is composed of a high level server, back end processing program, emergency report system and SQL database. After receiving the emergency signal and biomedical information, the processing plot is shown as in Figure 2.

\section{First Aid Clients}

The First Aid circuits are divided into four parts, first aid unit, medical unit, family unit, and mobile care unit. After the server receives the signal, it will inform the associated units:

1) First Aid unit: The server will provide the position and health information to these units. Such as the fire control unit emergency care unit.

2) Medical unit: The local hospital can contact the emergency care unit to access the biomedical information of the user.

3) Family: It will contact the user's requested family member. If the system has not contacted other units for any reason, the family member can contact assistance by another medium.

4) Mobile care unit: Ambulance, for an example, can access the user's position and biomedical information from the server.

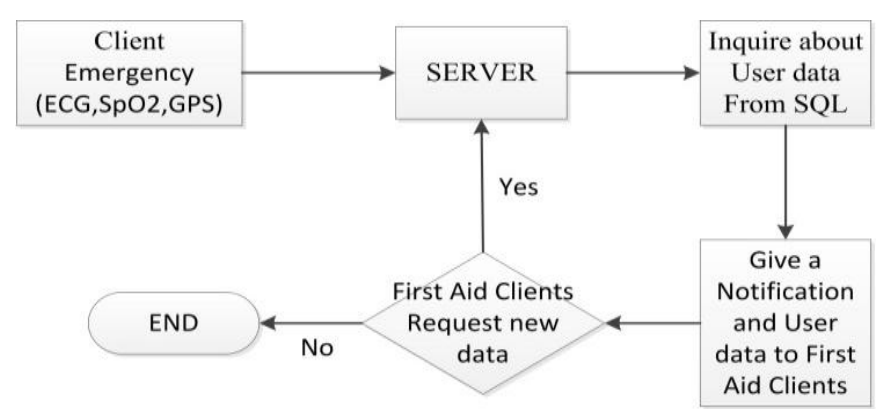

Fig. 2. Server Side System

\section{HARDWARE}

The whole hardware system, not only the server predominantly the user devices, includes BAD and other communicating devices:

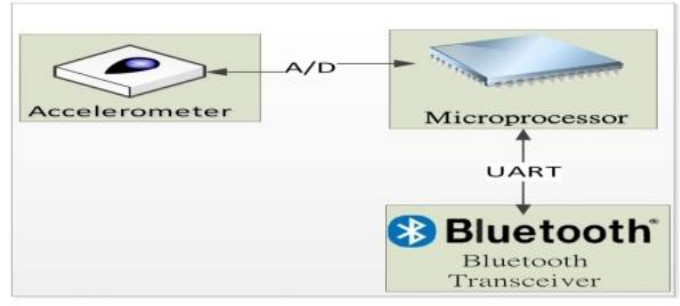

Fig. 3. BAD Hardware Architecture

\section{A. BAD Device}

Figure 3 details the block diagram of the BAD hardware architecture. The BAD is mainly built from a microprocessor, an accelerometer, Bluetooth transceiver and other elements. Its function is to detect the body's reaction to signals, abnormal analysis, and the control of signal transfer and receive.

Explanations for each device are listed below:

1) Microprocessor: SCI4431 [12] is used as the controller of BAD in this research. SCI4431 contains a RISC (Reduced Instruction Set Computer) micro controller, DSP (Digital Signal Processor), and PCM (Pulse Code Modulation) codec. The micro controller is used to operate the Bluetooth protocol stack, and analyze the accelerometer signals. DSP and PCM codec are used for sound signal operation and decoding.

2) Accelerometer: A MXA2500 Dual Axis accelerometer [13]is used in this project. MXA2500 is an electro-mechanical integrated device which can be used to detect the changes in acceleration. The change of acceleration is a control point of the body condition in this research. The MXA2500 communicates with the microprocessor through the $A / D$ (Analog/Digital) interface.

3) Bluetooth Transceiver: iWRAP [14] is used as the baseband IC of the Bluetooth transceiver, which communicates with the microprocessor through an UART interface.

\section{B. Communication Devices}

Figure 4, represents the Bluetooth composed Pico-Net [15]. It forms communication devices by operating SPP. 




Fig. 4. Bluetooth Pico-Net

Figure 5 presents the system communication plot. BAD, ECG and $\mathrm{SpO} 2$ will shut down the system into a power save mode after Bluetooth and mobile phone pairing. When the $\mathrm{BAD}$ detects an emergency, it will resume normal power, and contact the mobile phone to call out ECG and $\mathrm{SpO}_{2}$ processing.

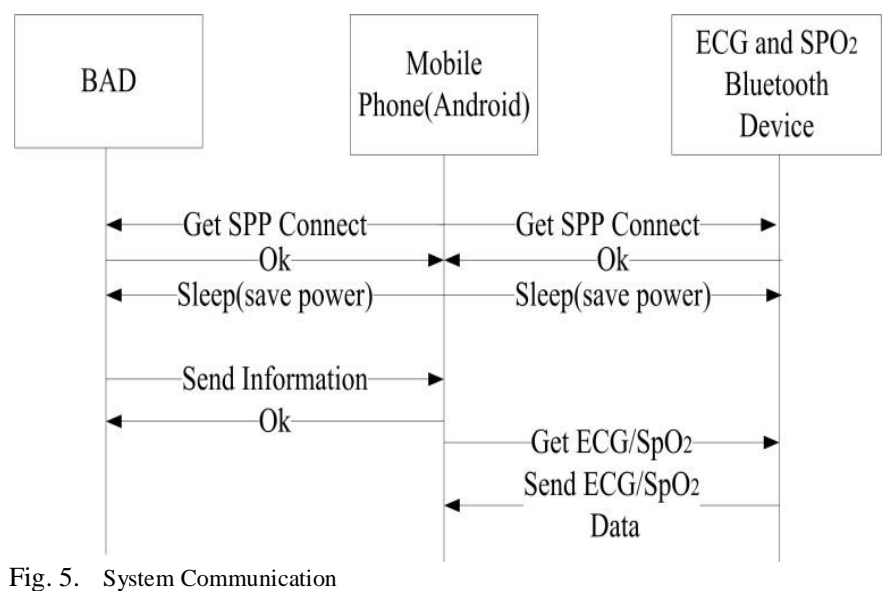

IV. SOFTWARE

This part of the software design includes BAD motion editing and its analysis process. When the system is told to run into the part of emergency procedure, the processing details like before:

\section{A. $B A D$}

BAD in this research contains two different modes according to different body conditions, namely the body stimulation mode and body activity detection mode [15]. The details of which are as follows:

\section{1) Body Stress-Reaction Mode Signal Processing}

2) Body Activity Detection Mode Signal Processing

This mode is opposite to the body stress-reaction mode, which means it detects the body activity changing from the normal level to a level of low activity. It represents that something wrong might have happened, and BAD immediately starts the emergency procedures. A microprocessor reads the signal from the accelerometer every 10 milliseconds, and passes the signal through one order passive HPF, and then calculates the RMS. If the RMS is lower than the preset static RMS threshold for a period of time, it means that an emergency has occurred. The system would beep out the warning sound in the first instance, and if the user does not turn off the alarm, the system would start emergency procedures.

\section{B. Emergency procedure}

After receiving the BAD emergency signal, the system will enter the emergency procedure mode, as shown in Figure 6. Firstly, it will recheck the signal. If it is still an emergency signal, it would start the GPS to determine where the user is and identify whether the target moves. If the target stays still, it will start the ECG and $\mathrm{SpO}_{2}$ Bluetooth devices. After receiving the data, it processes mobile phone and monitoring and displaying and alerts nearby people for further assistance. As the same time, the data is sent back to the server which also informs the First Aid Units to send out further help.

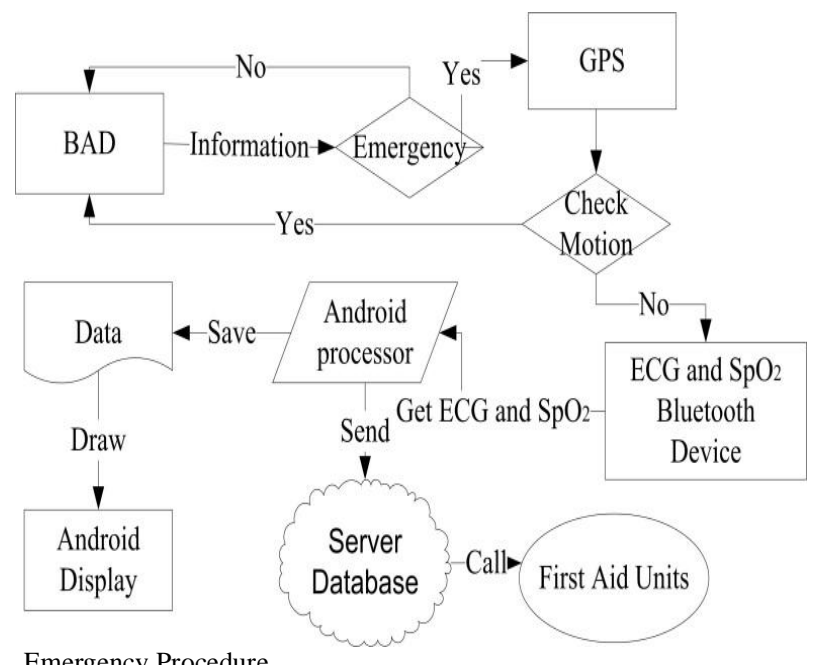

Fig. 6. Emergency Procedure

\section{Measurement Results}

After the Android phone receives the signal from BAD and enters the emergency procedure mode, the Android phone will start receiving an ECG signal. For receiving the ECG signal we used a Bluetooth class of Android mobile phone to receive the ECG data with a 16 bit microchip.

This chip edits two groups of 8 bits data and uses a SPP profile to transfer data. Finally it converts the ECG data using formula (1) to determine the value, which is the ECG detection value.

$$
\mathrm{ECG}=\mid(\text { Data }[0] \mathrm{X} 256+\text { Data [1] }) \mathrm{X} 32.23 \mid \div 10000
$$

When we receive the data, we convert these data to the signal shown in Figure 7. Upon standing up, both psychology and physiology conditions of the user become normal as shown in Figure 8.

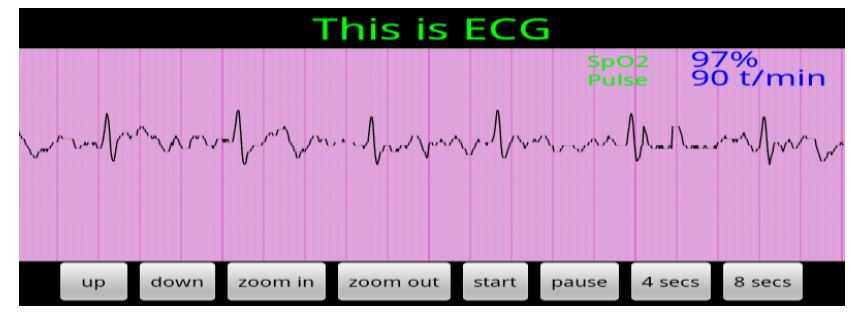

Fig. 7. Fall down status 


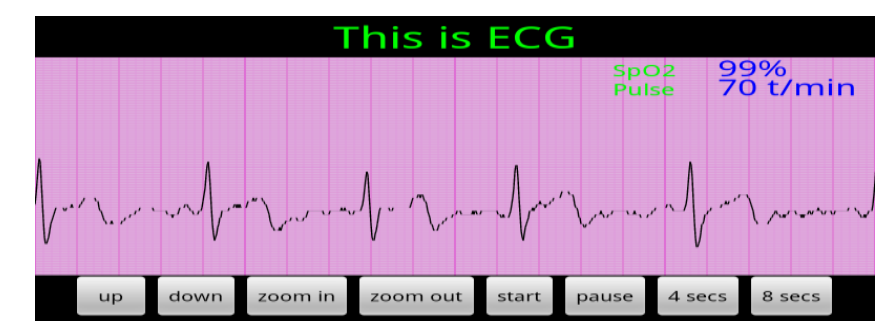

Fig. 8. Normal status

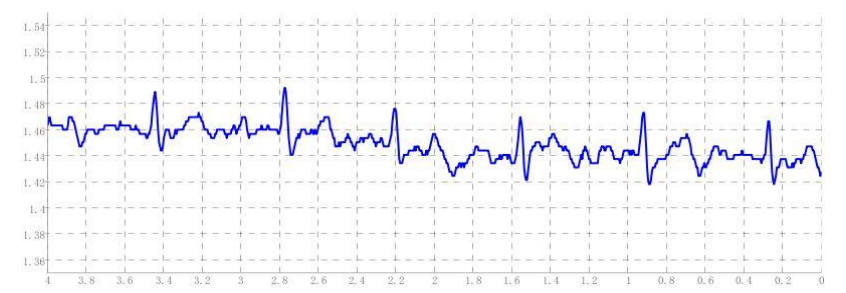

Fig. 9. Server Web-End

The remote and other members through the WEB interface confirm the biomedical information such as shown in Figure 9, the server and receives signal when simulating people falling down. At the same time, it uses GPS to confirm the client's location, for an efficient response time, and sends an ambulance to help. Strives see the Figure 10.

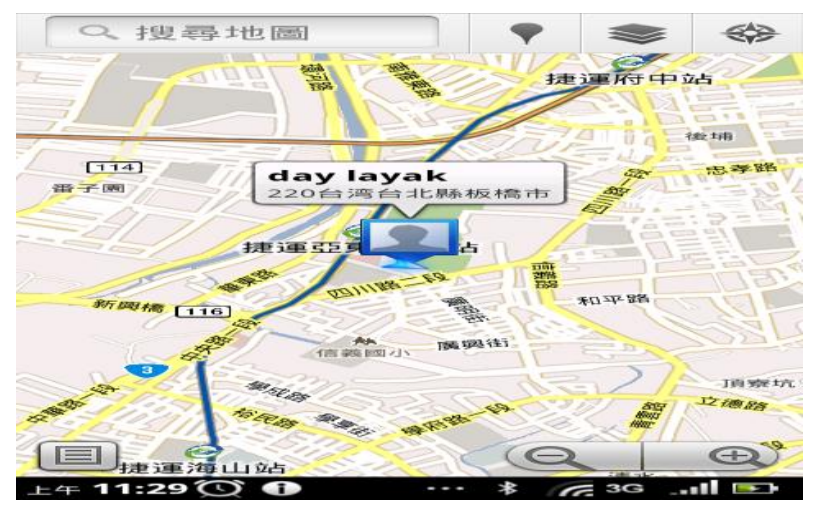

Fig. 10. GPS Location

\section{CONCLUSION}

To develop an Android mobile phone transfer function, the proposed design with the fall down alarm and GPS functions provides a simple, practical and portable system of real-time monitoring of human body activity by BAD, ECG, pulsimeter, $\mathrm{SpO} 2$, or other future developed biomedical devices. These devices detects the user being in both a static and dynamic emergency by separate body stress-reaction mode and body activity detection mode, and suggests a solution with ECG, pulsimeter and $\mathrm{SpO} 2$, or others, in the emergency situation. In particular this is designed for the elderly and patients who are at risk; we expect that people who carried this device would have more effective care and assistance from others.

\section{REFERENCES}

[1] Cummins RO, Eisenberg MS, Hallstrom AP, Litwin PE. "Survival of out-of-hospital cardiac arrest with early initiation of cardiopulmonary resuscitation", Am J Emerg Med. pp.114-pp.119, 1985.

[2] Albarede JL, Lemieux A, Vellas B, Groulx B. "Psychological factors in falls in elderly patients", Can J Psychiat ; 34(2):94-6,1989.

[3] S. Dagtas, G. Pekhteryev, Z. S, ahinoglu, H. Cam, and N. Challa."RealTime and Secure Wireless Health Monitoring", International Journal of Telemedicine and Applications, 2008.

[4] Stanford, V. "Pervasive health care applications face tough security challenges", IEEE Pervasive Computing, April-June, Vol 1, Issue: 2, pp 8-pp12, 2002.

[5] Sultan S., Mohan P., Sultan N, "Experiences from a Newe-Health Initiative for Patients with Diabetes and Cardiovascular Disease", In 1st IEEE International WoWMoM Workshop on Interdisciplinary Research on E-Health Services and Systems. Kos, Greece, June, 2009.

[6] Ren-Guey Lee, Kuei-Chien Chen, Chun-Chieh Hsiao, and ChwanLuTseng,"A Mobile Care System With Alarm Mechanism”, IEEE Transactions on Information Technology in Biomedicine, vol.11, no.5, September, 2007

[7] Maki BE, Holliday PJ, Topper AK. Fear of falling and postural performance in the elderly J Gerontol; M123-31, 1991.

[8] Bluetooth Specification, "Serial Port Profile", Version 1.1, February,2001.

[9] U.Anliker, at el.“Amon:A wearable multi-parameter medical monitoring and alarm system",IEEE Transactions on Information Technology in Biomedicine, pp415-pp427, 2004.

[10] Foad Dabiri, Tammara Massey, Hyduke Noshadi, Hadop Hagopian, C.K.Lin, Robert Tan, Jocob Schmidt, and Majid Sarrafzadeh,"A Telehealth Architecture for Networked Embedded Systems: A Case Study in Vivo Health Monitoring" ,IEEE Transactions on Information Technology in Biomedicine, vol. 13, no. 3, pp351-pp359, May, 2009.

[11] Gotop Information Inc., The experiment of Google Android 2.X Application program development, , October ,2010.

[12] National Semiconductor Inc., "SC14440/431/432/435/436 /437/438. Baseband Processor for PP/FP DECT and WDCT", VI.1, June 16, 2005.

[13] Memsic Inc.,"MXA2500G/M Improved Ultra Low Noise \pm 1.7 g Dual Axis Accelerometer with Absolute Outputs", Rev. G, 3/6/2007

[14] Bluegiga Technologies Inc. "iWRAP ${ }^{3}$ User Guide", 2008

[15] Ching-Sung Wang, Chien-Wei Liu, Teng-Wei Wang, "Tele-care for emergency announcements "J. Biomedical Science and Engineering, vol.3, no.8, pp.822-pp.827, 2010. 